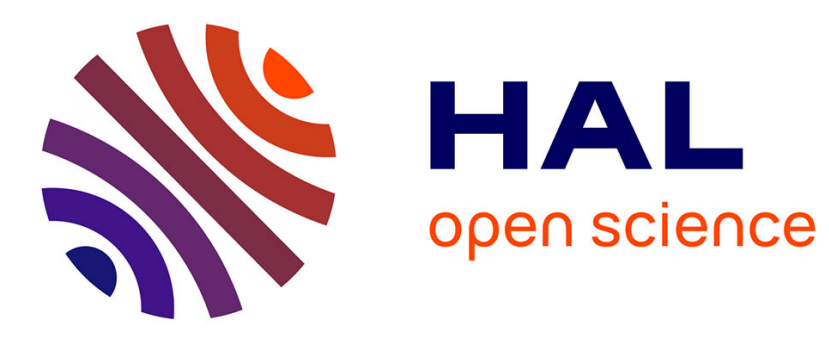

\title{
A model of influence in a social network
}

\author{
Michel Grabisch, Agnieszka Rusinowska
}

\section{To cite this version:}

Michel Grabisch, Agnieszka Rusinowska. A model of influence in a social network. Theory and Decision, 2010, 69 (1), pp.69-96. 10.1007/s11238-008-9109-z . halshs-00308741v2

\section{HAL Id: halshs-00308741 \\ https://shs.hal.science/halshs-00308741v2}

Submitted on 30 Jun 2010

HAL is a multi-disciplinary open access archive for the deposit and dissemination of scientific research documents, whether they are published or not. The documents may come from teaching and research institutions in France or abroad, or from public or private research centers.
L'archive ouverte pluridisciplinaire HAL, est destinée au dépôt et à la diffusion de documents scientifiques de niveau recherche, publiés ou non, émanant des établissements d'enseignement et de recherche français ou étrangers, des laboratoires publics ou privés. 


\title{
A model of influence in a social network
}

\author{
MICHEL GRABISCH ${ }^{1}$ and AGNIESZKA RUSINOWSKA ${ }^{2}$ \\ 1 Université Paris I Panthéon-Sorbonne \\ Centre d'Economie de la Sorbonne, 106-112 Bd de l'Hôpital, 75013 Paris, France \\ Tel (+33) 144078 285, Fax (+33) 144078301 \\ michel.grabisch@univ-paris1.fr \\ 2 GATE, CNRS UMR 5824 - Université Lumière Lyon 2 - Ecole Normale Supérieure LSH \\ 93, Chemin des Mouilles - B.P.167, 69131 Ecully Cedex, France \\ Tel (+33) 472866080 , Fax $(+33) 472866090$ \\ rusinowska@gate.cnrs.fr
}

\begin{abstract}
In the paper, we study a model of influence in a social network. It is assumed that each player has an inclination to say YES or NO which, due to influence of other players, may be different from the decision of the player. The point of departure here is the concept of the Hoede-Bakker index - the notion which computes the overall decisional 'power' of a player in a social network. The main drawback of the Hoede-Bakker index is that it hides the actual role of the influence function, analyzing only the final decision in terms of success and failure. In this paper, we separate the influence part from the group decision part, and focus on the description and analysis of the influence part. We propose among other descriptive tools a definition of a (weighted) influence index of a coalition upon an individual. Moreover, we consider different influence functions representative of commonly encountered situations. Finally, we propose a suitable definition of a modified decisional power.
\end{abstract}

JEL Classification: C7, D7

Keywords: influence function, influence index, decisional power, social network 


\section{Introduction}

A considerable part of the literature in social choice and voting games concerns power indices and related concepts. There is a vast literature which presents a theoretical analysis of power indices, their axiomatization as well as their applications; see e.g., Penrose (1946), Banzhaf (1965), Rae (1969), Shapley and Shubik (1954), Dubey and Shapley (1979), Coleman (1971, 1986), Deegan and Packel (1978), Holler and Packel (1983), Johnston (1978), van den Brink and van der Laan (1998), van der Laan and van den Brink (1998, 2002), Felsenthal et al. (1998); see e.g., Felsenthal and Machover (1998) for an overview.

In the literature, also some measures of 'power' or 'control' in networks have been proposed. For a network, which consists of a set of positions and a set of bilateral links between the positions, one may define a cooperative transferable utility game which measures the power of coalitions of positions and, for instance, one may apply the Shapley value (Shapley, 1953) to this network power game; see e.g., van den Brink and Gilles (2000), van den Brink and Borm (2002), van den Brink et al. (2007).

One may also find in the literature various generalizations and extensions of the concept of power index. We may cite among them Owen (1977, 1981), Felsenthal and Machover (2002), and Feix et al. (2007), who consider a priori unions, blocs and alliances. In particular, the concept of alliance considers an internal mechanism among players of an alliance, who have a priori different opinions, but agree on a common decision. The question is then to study the power of the alliance compared to the power of the individuals in the alliance. In Hu and Shapley (2003), where a complex organization is presented with different kinds of players (boss, free agent, cog, yesman, etc.), the authors prefer to speak of an authority distribution among players rather than of a power index.

Taking a more general point of view, this kind of situations may be viewed as instances of influence among players, in the sense that their final decision may differ from their original inclination. Specifically, it is considered that each player has an inclination (original decision) to say YES or NO in some voting situation. For each possible configuration $i$ of individual inclinations, it is supposed that after mutual influence the actual decision $B i$ of all players is made, where $B$ denotes the influence function. Then, a group decision $g d(B i)$ is made. This is in fact the framework considered originally in Hoede and Bakker (1982), who proposed the concept of decisional power of a player (the Hoede-Bakker index), and which was later generalized and modified in Rusinowska and De Swart (2006).

Despite the interest of the idea of decisional power of a player in a situation where influence exists, the Hoede-Bakker index fails to give a full description of the situation, in the sense that it hides the actual role of the influence function $B$, analyzing only the final decision in terms of success and failure w.r.t. the original inclination. To the best of our knowledge, there is no specific work which focuses on the influence phenomenon and tries to describe and analyze it. This is precisely what we aim for in this paper. More precisely, our aims are:

- to introduce indices measuring the influence among players in networks (Section 3);

- to provide tools describing and analyzing the influence function B (Section 4);

- to study properties of some specific influence functions (Section 5). 
In other words, we separate in our description the influence part and the group decision part. Since to our knowledge the former has not been studied per se, we focus on this part of the description, which constitutes the most original part of the paper and is presented in Sections 3, 4, and 5. Essentially, we propose the following tools:

- An index of influence of a coalition $S$ upon a player $i$ outside $S$. The general idea is to compute a weighted number of times the coalition $S$ makes player $i$ change his decision. Two particular ways of weighting lead to a possibility influence index which takes into account any possibility of influence, and to a certainty influence index which expresses certainty of influence. We also distinguish between a direct influence ( $i$ follows $S$ ), and opposite influence (by a kind of reactive behavior, $i$ decides the opposite of $S$ ).

- The concept of follower, where by a follower of a given coalition of players we mean the voter who always decides according to the inclination of the coalition in question. Analogously, we propose the concept of anti-follower for opposite influence.

- The concept of purely influential function of one coalition upon another coalition. Function $B$ is a purely influential function of coalition $S$ upon coalition $T$ if, in case all players of $S$ have the same inclination, all members of $T$ decide according to the inclination of $S$, and all players outside $T$ follow their own inclination. A purely influential function is called canonical if additionally, in case the players of $S$ do not have the same inclination, all players decide according to their own inclinations. Besides, we propose several typical examples of influence functions, depicting commonly encountered situations, such as a majority function, a 'guru' function, the identity function, and some others.

- The concept of kernel of an influence function, that is, the set of its 'true' (minimal) influential coalitions.

The structure of the paper is the following. In Section 2, we recall the original and the generalized Hoede-Bakker indices. In Section 3, the various influence indices are defined. The concepts of followers and purely influential functions are analyzed in Section 4. Different influence functions and their properties are studied in Section 5. The notions of success, decisiveness, and the modified decisional power are considered in Section 6 . Conclusions, including some ideas for future research, are presented in Section 7. All concepts introduced in the paper are illustrated with a three-player family example.

\section{The Hoede-Bakker index}

First we recapitulate the original Hoede-Bakker index as introduced in Hoede and Bakker (1982), and its generalization given in Rusinowska and De Swart (2006). The general framework is the following. We consider a social network with the set of all players (agents, actors, voters) denoted by $N:=\{1, \ldots, n\}$. The players have to make a certain acceptance-rejection decision. Each player has an inclination either to say YES (denoted by +1 ) or NO (denoted by -1 ). An inclination vector, denoted by $i$, is an $n$-vector consisting of ones and minus ones. Let $I:=\{-1,+1\}^{n}$ be the set of all inclination vectors. For convenience, $(1,1, \ldots, 1) \in I$ is denoted by $1_{N}$, similarly for $-1_{N}$, and also for mixed cases like $\left(-1_{N \backslash S}, 1_{S}\right)$.

It is assumed that players may influence each others, and due to the influences in the network, the final decision of a player may be different from his original inclination. 
In other words, each inclination vector $i \in I$ is transformed into a decision vector $B i$, where $B: I \rightarrow I, i \mapsto B i$ is the influence function. The coordinates of $B i$ are denoted by $(B i)_{k}, k \in N$. The set of all influence functions will be denoted by $\mathcal{B}$. The decision vector $B i$ is an $n$-vector consisting of ones and minus ones and indicating the decisions made by all players. Let $B(I)$ be the set of all decision vectors under $B$. Furthermore, the group decision function $g d: B(I) \rightarrow\{+1,-1\}$ is introduced, having the value +1 if the group decision is YES, and the value -1 if the group decision is NO. The set of all group decision functions will be denoted by $\mathcal{G}$. The following definition has been introduced in Hoede and Bakker (1982):

Definition 1 Given $B \in \mathcal{B}$ and $g d \in \mathcal{G}$, the decisional power (the Hoede-Bakker index) of a player $k \in N$ is given by

$$
\operatorname{HB}_{k}(B, g d):=\frac{1}{2^{n-1}} \sum_{\left\{i \mid i_{k}=+1\right\}} g d(B i) .
$$

In Rusinowska and De Swart (2006), a certain generalization of the Hoede-Bakker index has been proposed:

Definition 2 Given $B \in \mathcal{B}$ and $g d \in \mathcal{G}$, the generalized Hoede-Bakker index of a player $k \in N$ is given by

$$
\operatorname{GHB}_{k}(B, g d):=\frac{1}{2^{n}}\left(\sum_{\left\{i \mid i_{k}=+1\right\}} g d(B i)-\sum_{\left\{i \mid i_{k}=-1\right\}} g d(B i)\right) .
$$

Although the (generalized) Hoede-Bakker index is defined in the framework of influence, this is not a measure of influence among players. First of all, we notice that neither in the original definition of the Hoede-Bakker index nor in its generalization mentioned above, the functions $B$ and $g d$ are considered separately. When calculating the (original or generalized) Hoede-Bakker index, only the relation between an inclination vector $i$ and the group decision $g d(B i)$ is taken into account. Although incorporating an influence aspect into the framework could be seen as an attractive feature of the Hoede-Bakker index, in fact the actual role of the influence function $B$ is hidden. In order to see this better, let us consider the following example.

Example 1 All the concepts introduced in the paper will be illustrated in a leading example, which will be referred to as the Family Example. We analyze a three-actor family network with a child (player 1), a mother (player 2) and a father (player 3). The family has to decide for a long Sunday bicycle trip. The child is influenced by his parents in the sense that if the parents have the same inclinations, the child will follow them, but if their inclinations differ from each other, the child will decide according to his own inclination. It is a 'fair' family decision-making, and the majority vote is assumed: the family decides for the trip if at least two family members say YES. Table 1 presents the group decision for the example.

By virtue of (1), we get the following:

$$
\operatorname{GHB}_{1}(B, g d)=\operatorname{GHB}_{2}(B, g d)=\operatorname{GHB}_{3}(B, g d)=\frac{1}{2} .
$$


Table 1. The group decision

\begin{tabular}{c|c|c|c|c|c|c|c|c}
$i \in I$ & $(1,1,1)$ & $(1,1,-1)$ & $(1,-1,1)$ & $(-1,1,1)$ & $(1,-1,-1)$ & $(-1,1,-1)$ & $(-1,-1,1)$ & $(-1,-1,-1)$ \\
\hline$B(i)$ & $(1,1,1)$ & $(1,1,-1)$ & $(1,-1,1)$ & $(1,1,1)$ & $(-1,-1,-1)$ & $(-1,1,-1)$ & $(-1,-1,1)$ & $(-1,-1,-1)$ \\
\hline$g d(B i)$ & 1 & 1 & 1 & 1 & -1 & -1 & -1 & -1
\end{tabular}

Although no influence of the child on the parents is assumed here, the index of player 1 is equal to the indices of the remaining (influencing) players. Consequently, one can see a need to introduce an index which indeed measures an influence among players.

\section{The influence indices}

When analyzing the 'influence part' of the model, a question appears how to measure a degree of influence of a player (or a coalition) on the other voter(s). The answer is not that straightforward if we can just observe inclinations and final decisions of the players in a multi-player social network. Suppose the final decision of player A is different from his inclination, but this decision coincides with the inclinations of two other players in the network, say, agents B and C. Is voter A's decision different from his inclination because of the unique influence of player $\mathrm{B}$, or the unique influence of player $\mathrm{C}$, or maybe A has decided differently from his inclination only because he has faced an influence of the strong two-party coalition? These are the questions that not always can be answered univocally if apart from knowing the function $B$, we are not able to observe a 'real act of influencing among players'. Consequently, we introduce a family of influence indices.

Generally speaking, we say that a player is influenced by a coalition (of players with the same inclination) if the decision of the player is different from his (original) inclination. Such a general definition of influence can be motivated as follows. Since the player has 'changed his mind' and decided differently from his preliminary thoughts, there had to be a kind of influence from 'outside' which has caused this change. We assume this 'outside' influence to come from other agents in the network who are unanimous in their opinions (inclinations). We distinguish between a direct influence and an opposite influence. In the first case, the player's inclination is different from the inclination of that coalition, but the player's decision is the same as the inclination of the coalition. In the latter case, the player's inclination coincides with the inclination of the coalition, but by a kind of reactive behavior, his decision is different from the inclination of the coalition. The direct influence of a coalition is therefore related to the ability to make the players, who are inclined differently from the coalition, decide according to the inclination of that coalition. The concept of the opposite influence captures, in particular, the case of an 'independent child': although his inclination coincides with the parents' inclination, in order to show his 'independence', he decides differently from his parents' inclination, and consequently, differently from his own inclination.

Of course, the direct influence and the opposite influence are related to each other, in the sense that usually there are both the direct influence and the opposite influence, coming from different coalitions. Suppose there is a coalition with the positive inclination, another coalition with the negative inclination, and a player who has the inclination to say NO, but finally he says YES. In such a situation, we record both the direct influence of the first coalition, and the opposite influence of the latter coalition on the given player, 
but we cannot know the actual reason for which the player has decided differently from his inclination. Since these two kinds of influence are related to each other, and since in principle the direct influence seems to be more 'natural' than the opposite influence, in some sections we present results which concern the direct influence only.

Before formalizing the influence concepts, we introduce several notations for convenience. First of all, cardinality of sets $S, T, \ldots$ will be denoted by the corresponding lower case $s, t, \ldots$ We omit braces for sets, e.g., $\{k, m\}, N \backslash\{j\}, S \cup\{j\}$ will be written $k m$, $N \backslash j, S \cup j$, etc. We also introduce for any $S \subseteq N,|S| \geq 2$, the set $I_{S}$ of all inclination vectors under which all members of $S$ have the same inclination

$$
I_{S}:=\left\{i \in I \mid \forall k, j \in S\left[i_{k}=i_{j}\right]\right\},
$$

and $I_{k}:=I$, for any $k \in N$. We denote by $i_{S}$ the value $i_{k}$ for some $k \in S, i \in I_{S}$. Let for each $S \subseteq N$ and $j \in N$

$$
\begin{gathered}
I_{S \rightarrow j}:=\left\{i \in I_{S} \mid i_{j}=-i_{S}\right\} \\
I_{S \rightarrow j}^{*}(B):=\left\{i \in I_{S \rightarrow j} \mid(B i)_{j}=i_{S}\right\} .
\end{gathered}
$$

$I_{S \rightarrow j}$ and $I_{S \rightarrow j}^{*}(B)$ denote the set of all inclination vectors of potential direct influence of $S$ on $j$, and the set of all inclination vectors of direct influence of $S$ on $j$ under given $B \in \mathcal{B}$, respectively. Similarly, we introduce the set of all inclination vectors of potential opposite influence and the set of all inclination vectors of opposite influence of a coalition on a player. Let for each $S \subseteq N$ and $j \in N$

$$
\begin{gathered}
I_{S \rightarrow j}^{o p}:=\left\{i \in I_{S} \mid i_{j}=i_{S}\right\} \\
I_{S \rightarrow j}^{* o p}(B):=\left\{i \in I_{S \rightarrow j}^{o p} \mid(B i)_{j}=-i_{S}\right\} .
\end{gathered}
$$

Some elementary properties of these notions are given below.

Proposition 1 Let $\emptyset \neq S, S^{\prime} \subseteq N$, with $S \subseteq S^{\prime}$, and $B \in \mathcal{B}$. The following holds.

(i) $I_{S \rightarrow j}=I_{S \rightarrow j}^{*}(B)=\emptyset$ for each $j \in S$.

(ii) $I_{S \rightarrow j}^{o p}=I_{S}$, for each $j \in S$.

(iii) $\left|I_{S \rightarrow j}\right|=\left|I_{S \rightarrow j}^{o p}\right|=2^{n-s}$, for each $j \in N \backslash S$.

(iv) $I_{S \rightarrow j} \cup I_{S \rightarrow j}^{o p}=I_{S}$, for each $j \in N$.

(v) $I_{S} \supseteq I_{S^{\prime}}, I_{S \rightarrow j} \supseteq I_{S^{\prime} \rightarrow j}, I_{S \rightarrow j}^{o p} \supseteq I_{S^{\prime} \rightarrow j}^{o p}$, for each $j \in N$.

(vi) $I_{S \rightarrow j}^{*}(B) \supseteq I_{S^{\prime} \rightarrow j}^{*}(B), I_{S \rightarrow j}^{* o p}(B) \supseteq I_{S^{\prime} \rightarrow j}^{* 0 p}(B)$, for each $j \in N$.

\subsection{The 'basic' influence indices}

Below, we formalize the influence concepts. First, we introduce the possibility influence indices.

Definition 3 Given $B \in \mathcal{B}$, for each $S \subseteq N$ and $j \in N \backslash S$, the possibility direct influence index of coalition $S$ on player $j$ is defined as

$$
\bar{d}(B, S \rightarrow j):=\frac{\left|I_{S \rightarrow j}^{*}(B)\right|}{\left|I_{S \rightarrow j}\right|}
$$

and the possibility opposite influence index of coalition $S$ on player $j$ is defined as

$$
\bar{d}^{o p}(B, S \rightarrow j):=\frac{\left|I_{S \rightarrow j}^{* o p}(B)\right|}{\left|I_{S \rightarrow j}^{o p}\right|} .
$$


$\bar{d}(B, S \rightarrow j) \in[0,1]$ (respectively $\left.\bar{d}^{o p}(B, S \rightarrow j) \in[0,1]\right)$ measures a degree of a direct (respectively opposite) influence coalition $S$ has on player $j$, taking into account any possibility of influence. We check therefore how many inclination vectors of potential direct (respectively opposite) influence of coalition $S$ on player $j$ are indeed the vectors of direct (respectively opposite) influence of $S$ on $j$. We do not verify here the inclinations of the players outside $S \cup j$. In particular, a situation in which all players in $N \backslash(S \cup j)$ have the inclination different from the inclination of $S$, and each situation in which there are players in $N \backslash(S \cup j)$ with the same inclination as the inclination of $S$, are treated equally.

Switching to another extreme way of calculating influence degree gives us the definitions of the certainty influence indices. Here, we take into account only those situations in which all players outside $S \cup j$ have the inclination different from the inclination of $S$.

Definition 4 Given $B \in \mathcal{B}$, for each $S \subseteq N$ and $j \in N \backslash S$, the certainty direct influence index of coalition $S$ on player $j$ is given by

$$
\underline{d}(B, S \rightarrow j):=\frac{\left|\left\{i \in I_{S \rightarrow j}^{*}(B) \mid \forall p \notin S\left[i_{p}=-i_{S}\right]\right\}\right|}{2}
$$

and the certainty opposite influence index of coalition $S$ on player $j$ is given by

$$
\underline{d}^{o p}(B, S \rightarrow j):=\frac{\left|\left\{i \in I_{S \rightarrow j}^{* o p}(B) \mid \forall p \notin S \cup j\left[i_{p}=-i_{S}\right]\right\}\right|}{2} .
$$

$\underline{d}(B, S \rightarrow j) \in\left\{0, \frac{1}{2}, 1\right\}$ (respectively $\left.\underline{d}^{o p}(B, S \rightarrow j) \in\left\{0, \frac{1}{2}, 1\right\}\right)$ measures a degree of a certain (since only $S$ could have influenced $j$ ) direct (respectively opposite) influence coalition $S$ has on player $j$. Note that for each $S \subseteq N$ and $j \in N \backslash S$

$$
\underline{d}^{o p}(B, S \rightarrow j)=\underline{d}(B, N \backslash(S \cup j) \rightarrow j) .
$$

\subsection{The weighted influence indices}

Finally, we propose more general definitions of the influence indices, which we call the weighted influence indices. These indices take into account the number of agents with the same inclination as the inclination of an influencing coalition. The main idea of the weighted index is therefore to capture different alternatives for 'sharing the influence ability' between all agents with the same inclination as the inclination of the coalition in question. The weighted indices capture, in particular, the two basic cases presented in Section 3.1.

Let for each $S \subseteq N, j \notin S$ and $i \in I_{S}$

$$
n^{*}(S, j, i):=\left|\left\{m \in N \backslash j \mid i_{m}=i_{S}\right\}\right| \geq s .
$$

$n^{*}(S, j, i)$ is the number of players with the same inclination as players of $S$ under $i \in I_{S}$ (including the players from $S$, but excluding player $j$ ).

For each $S \subseteq N, j \in N \backslash S$ and $i \in I_{S}$, we introduce a weight $\alpha_{i}^{S \rightarrow j} \in[0,1]$ of influence of coalition $S$ on $j \in N \backslash S$ under the inclination vector $i \in I_{S}$. We assume that for each $S \subseteq N$ and $j \in N \backslash S$, there exists $i \in I_{S \rightarrow j}$ such that $\alpha_{i}^{S \rightarrow j}>0$, and there exists $i \in I_{S \rightarrow j}^{o p}$ 
such that $\alpha_{i}^{S \rightarrow j}>0$. Moreover, we impose the symmetry assumption that $\alpha_{i}^{S \rightarrow j}$ depends solely on $n^{*}(S, j, i)$. Hence, one may note that for each $\emptyset \neq S \subseteq N$ and $j \in N \backslash S$

$$
\sum_{i \in I_{S \rightarrow j}^{+}} \alpha_{i}^{S \rightarrow j}=\sum_{i \in I_{S \rightarrow j}^{-}} \alpha_{i}^{S \rightarrow j},
$$

with

$$
I_{S \rightarrow j}^{+}:=\left\{i \in I_{S \rightarrow j} \mid i_{S}=+1\right\}, \quad I_{S \rightarrow j}^{-}:=\left\{i \in I_{S \rightarrow j} \mid i_{S}=-1\right\} .
$$

Remark also that

$$
\sum_{i \in I_{S \rightarrow j}} \alpha_{i}^{S \rightarrow j}=\sum_{i \in I_{S \rightarrow j}^{o p}} \alpha_{i}^{S \rightarrow j}
$$

Definition 5 Given $B \in \mathcal{B}$, for each $S \subseteq N, j \in N \backslash S$, the weighted direct influence index of coalition $S$ on player $j$ is defined as

$$
d_{\alpha}(B, S \rightarrow j):=\frac{\sum_{i \in I_{S \rightarrow j}^{*}(B)} \alpha_{i}^{S \rightarrow j}}{\sum_{i \in I_{S \rightarrow j}} \alpha_{i}^{S \rightarrow j}} \in[0,1]
$$

and the weighted opposite influence index of coalition $S$ on player $j$ is defined as

$$
d_{\alpha}^{o p}(B, S \rightarrow j):=\frac{\sum_{i \in I_{S \rightarrow j}^{* o p}(B)} \alpha_{i}^{S \rightarrow j}}{\sum_{i \in I_{S \rightarrow j}^{o p}} \alpha_{i}^{S \rightarrow j}} \in[0,1] .
$$

The possibility and certainty influence indices are recovered as follows. For each $S \subseteq N$, $j \in N \backslash S$ and $B \in \mathcal{B}$

$$
\begin{aligned}
\bar{d}(B, S \rightarrow j)= & d_{\bar{\alpha}}(B, S \rightarrow j), \quad \bar{d}^{o p}(B, S \rightarrow j)=d_{\bar{\alpha}}^{o p}(B, S \rightarrow j) \\
& \text { where } \bar{\alpha}_{i}^{S \rightarrow j}=1 \text { for each } i \in I_{S} \\
\underline{d}(B, S \rightarrow j)= & d_{\underline{\alpha}}(B, S \rightarrow j), \quad \underline{d}^{o p}(B, S \rightarrow j)=d_{\underline{\alpha}}^{o p}(B, S \rightarrow j)
\end{aligned}
$$

where for each $i \in I_{S}$

$$
\underline{\alpha}_{i}^{S \rightarrow j}= \begin{cases}1, & \text { if } \forall p \notin S \cup j, i_{p}=-i_{S} \\ 0, & \text { otherwise. }\end{cases}
$$

Hence, both in the possibility and certainty influence indices, the weight of influence is equal to 1 . The essential difference is that when calculating the possibility influence index, all possible cases of influence are treated equally, while for the certainty influence indices only the cases in which all players outside $S \cup j$ are inclined differently from $S$ are considered.

Apart from the possibility and certainty influence indices, we specify other ones which we denote by $d^{*}$ and $d^{* o p}$, and refer to as the equidistributed direct influence index and the equidistributed opposite influence index, respectively. Given $B \in \mathcal{B}$, for each $S \subseteq N$, $j \in N \backslash S$

$$
d^{*}(B, S \rightarrow j)=d_{\alpha^{*}}(B, S \rightarrow j), \quad d^{* o p}(B, S \rightarrow j)=d_{\alpha^{*}}^{o p}(B, S \rightarrow j)
$$




$$
\text { where } \alpha_{i}^{* S \rightarrow j}=\frac{1}{2^{n^{*}(S, j, i)}-1} \text { for each } i \in I_{S} \text {. }
$$

The definition of the equidistributed influence index can be explained as follows: for each $S \subseteq N, j \in N \backslash S$ and $i \in I_{S}$, we count the number of all $p$-player coalitions of the set $\left\{m \in N \backslash j \mid i_{m}=i_{S}\right\}$, where $p=1, \ldots, n^{*}(S, j, i)$, which gives

$$
\sum_{p=1}^{n^{*}(S, j, i)}\left(\begin{array}{c}
n^{*}(S, j, i) \\
p
\end{array}\right)=2^{n^{*}(S, j, i)}-1 .
$$

Hence, given $S$ and $j$, two inclination vectors with equal numbers of agents having the same inclination as the inclination of $S$ are treated equally, and the maximal weight 1 is divided by the number of possible coalitions that could be 'responsible' for the influence.

Finally, remark that the weighted influence index has no monotonicity property w.r.t. $S$, i.e., it is not true in general that if $S \subseteq S^{\prime}$, then $d_{\alpha}(B, S \rightarrow j) \leq d_{\alpha}\left(B, S^{\prime} \rightarrow j\right)$ or the converse. This is because both $I_{S \rightarrow j}$ and $I_{S \rightarrow j}^{*}(B)$ are antitone, so the behavior of their ratio is unpredictable.

\subsection{The Family Example continued}

We calculate the influence indices for the Family Example. Let us recall that

$$
N=\{1=\text { child }, 2=\text { mother, } 3=\text { father }\} .
$$

In this example, there is no opposite influence of a coalition on a player, but there is a direct influence on the child. We have for each $S \subseteq N, j \in N \backslash S, d_{\alpha}^{o p}(B, S \rightarrow j)=0$,

$$
\begin{gathered}
d_{\alpha}(B, 1 \rightarrow 2)=d_{\alpha}(B, 1 \rightarrow 3)=d_{\alpha}(B, 2 \rightarrow 3)=d_{\alpha}(B, 3 \rightarrow 2)=0 \\
d_{\alpha}(B, 12 \rightarrow 3)=d_{\alpha}(B, 13 \rightarrow 2)=0, \quad d_{\alpha}(B, 23 \rightarrow 1)=1 \\
\bar{d}(B, 2 \rightarrow 1)=\bar{d}(B, 3 \rightarrow 1)=\frac{1}{2} \\
\underline{d}(B, 2 \rightarrow 1)=\underline{d}(B, 3 \rightarrow 1)=0 \\
d^{*}(B, 2 \rightarrow 1)=d^{*}(B, 3 \rightarrow 1)=\frac{1}{4}
\end{gathered}
$$

Note that the weighted direct influence index of a coalition of the parents on the child is equal to 1 , while the weighted influence indices of the remaining two-player coalitions are equal to 0 .

\section{The followers and purely influential functions}

Before we focus on the influence functions $B \in \mathcal{B}$, let us remark properties of some related concepts. One of them is the concept of a follower of a given coalition, that is, a voter who always follows the inclination of the coalition in question. We also introduce the concept of an anti-follower of a coalition, that is, a voter whose decisions are always different from the inclination of that coalition. 
Definition 6 Let $\emptyset \neq S \subseteq N$ and $B \in \mathcal{B}$. The set of followers of $S$ under $B$ is defined as

$$
F_{B}(S):=\left\{j \in N \mid \forall i \in I_{S}\left[(B i)_{j}=i_{S}\right]\right\} .
$$

The set of anti-followers of $S$ under $B$ is defined as

$$
\bar{F}_{B}(S):=\left\{j \in N \mid \forall i \in I_{S}\left[(B i)_{j}=-i_{S}\right]\right\} .
$$

Letting $F_{B}(\emptyset):=\emptyset, F_{B}$ is a mapping from $2^{N}$ to $2^{N}$.

We remark that $F_{B}(S) \supseteq S$ does not hold in general.

Proposition 2 Let $B \in \mathcal{B}$. Then the following holds:

(i) Whenever $S \cap T=\emptyset, F_{B}(S) \cap F_{B}(T)=\emptyset$.

(ii) $F_{B}$ is an isotone function $\left(S \subseteq S^{\prime}\right.$ implies $\left.F_{B}(S) \subseteq F_{B}\left(S^{\prime}\right)\right)$.

Consequently, if $F_{B}(N)=\emptyset$, then $F_{B} \equiv \emptyset$.

(iii) For each $j \in F_{B}(S) \backslash S, d_{\alpha}(B, S \rightarrow j)=1, d_{\alpha}^{o p}(B, S \rightarrow j)=0$.

Proof: (i) Since $S \cap T=\emptyset, I_{S} \cap I_{T}$ strictly includes $I_{S \cup T}$, and there exists $i \in I_{S} \cap I_{T}$ such that $i_{S}=-i_{T}$. Hence, if $j \in F_{B}(S) \cap F_{B}(T)$, the equality $(B i)_{j}=i_{S}=i_{T}$ cannot hold for this $i$.

(ii) Take $S \subseteq S^{\prime}$ and $j \in F_{B}(S) . i \in I_{S^{\prime}}$ implies $i \in I_{S}$ by antitonicity, hence $(B i)_{j}=i_{S}$ by hypothesis, and since $i_{S}=i_{S^{\prime}}, j \in F_{B}\left(S^{\prime}\right)$.

(iii) Let $B \in \mathcal{B}, S \subset N, F_{B}(S) \neq \emptyset$, and $j \in F_{B}(S) \backslash S$. Hence, for each $i \in I_{S},(B i)_{j}=i_{S}$, and therefore $I_{S \rightarrow j}^{*}(B)=I_{S \rightarrow j}, I_{S \rightarrow j}^{* o p}(B)=\emptyset$, which means that $d_{\alpha}(B, S \rightarrow j)=1$, and $d_{\alpha}^{o p}(B, S \rightarrow j)=0$.

Note that the weighted direct influence index of a coalition on its follower outside the coalition is equal to 1 , while the weighted opposite influence index is equal to 0. Also remark that (i) and (ii) hold for $\bar{F}_{B}$ as well, and for (iii), the dual statement holds.

Assume $F_{B}$ is not identically the empty set. Then the kernel of $B$ is the following collection of sets:

$$
\mathcal{K}(B):=\left\{S \in 2^{N} \mid F_{B}(S) \neq \emptyset \text {, and } S^{\prime} \subset S \Rightarrow F_{B}\left(S^{\prime}\right)=\emptyset\right\} .
$$

The kernel is well defined due to isotonicity of $F_{B}$. It is the set of "true" influential coalitions.

Definition 7 Let $S, T$ be two disjoint non empty subsets of $N$. Function $B$ is said to be a purely influential function of $S$ upon $T$ if it satisfies for all $i \in I_{S}$ :

$$
(B i)_{j}= \begin{cases}i_{S}, & \text { if } j \in T \\ i_{j}, & \text { otherwise }\end{cases}
$$

The set of such functions is denoted by $\mathcal{B}_{S \rightarrow T}$. 
If all members of $S$ have the same inclination, then each player from $T$ follows $S$, and each agent outside $T$ decides according to his own inclination. Note that these functions are arbitrary on $I \backslash I_{S}$. It is easy to see that the cardinality of $\mathcal{B}_{S \rightarrow T}$ is

$$
\left|\mathcal{B}_{S \rightarrow T}\right|=2^{n\left(2^{s}-2\right) 2^{n-s}} .
$$

In each $\mathcal{B}_{S \rightarrow T}$, there are 3 particular members. The minimal one is such that $B i=-1_{N}$ for all $i \in I \backslash I_{S}$, the maximal one is such that $B i=1_{N}$ for all $i \in I \backslash I_{S}$. More interesting is the one which is the identity function on $I \backslash I_{S}$. We call it the canonical pure influential function of $S$ upon $T$, and we denote it by $B_{S \rightarrow T}$.

Proposition 3 presents some properties of the purely influential function of $S$ on $T$. First of all, all members of $S \cup T$, and only them, are the followers of $S$. Moreover, the weighted influence index of $S$ on a player outside $S$ under the purely influential function is either equal to 1 , if the player is a member of $T$, or equal to 0 , if the player does not belong to $S \cup T$. Formally, we can write as follows:

Proposition 3 Let $S, T$ be two disjoint non empty subsets of $N$. Then the following holds:

(i) For all $B \in \mathcal{B}_{S \rightarrow T}, F_{B}(S)=S \cup T, \bar{F}_{B}(S)=\emptyset$.

(ii) For each $B \in \mathcal{B}_{S \rightarrow T}$ and $j \in N \backslash S$, dop $(B, S \rightarrow j)=0$, and

$$
d_{\alpha}(B, S \rightarrow j)= \begin{cases}1, & \text { if } j \in T \\ 0, & \text { if } j \in N \backslash(S \cup T) .\end{cases}
$$

Proof: (i) Take $t \in S \cup T$. If $t \in T$, then for any $i \in I_{S},(B i)_{t}=i_{S}$. If $t \in S$, then for any $i \in I_{S},(B i)_{t}=i_{t}=i_{S}$. Hence $t \in F_{B}(S)$. On the other hand, take $t \in F_{B}(S)$. Then for any $i \in I_{S},(B i)_{t}=i_{S}$, and hence $t \in S \cup T$. Now suppose $j \in \bar{F}_{B}(S)$. Hence, for each $i \in I_{S},(B i)_{j}=-i_{S}$, and therefore $j \notin S \cup T$ since $B \in \mathcal{B}_{S \rightarrow T}$. Let us assume $j \notin S \cup T$. If we take $i \in I_{S}$ such that $i_{j}=i_{S}$, we get $(B i)_{j}=i_{j}=i_{S}$, a contradiction.

(ii) Let $B \in \mathcal{B}_{S \rightarrow T}$. Then for each $i \in I_{S},(B i)_{j}=i_{S}$ for $j \in T$, and $(B i)_{j}=i_{j}$ for $j \notin T$. Since $I_{S \rightarrow j}^{o p} \subset I_{S}$, we have for each $i \in I_{S \rightarrow j}^{o p},(B i)_{j}=i_{S}$. Hence, $I_{S \rightarrow j}^{* o p}(B)=\emptyset$ which gives $d_{\alpha}^{o p}(B, S \rightarrow j)=0$. Moreover, since $I_{S \rightarrow j} \subset I_{S}$, we have for each $i \in I_{S \rightarrow j},(B i)_{j}=i_{S}$ for $j \in T$, and $(B i)_{j}=i_{j}$ for $j \notin T$. Hence, $I_{S \rightarrow j}^{*}(B)=I_{S \rightarrow j}$ for $j \in T$, and $I_{S \rightarrow j}^{*}(B)=\emptyset$ for $j \in N \backslash(S \cup T)$, and therefore if $j \in T$, then $d_{\alpha}(B, S \rightarrow j)=1$, and if $j \in N \backslash(S \cup T)$, then $d_{\alpha}(B, S \rightarrow j)=0$.

Example 2 The sets of followers and anti-followers of each coalition for the Family Example are the following:

$$
\begin{gathered}
F_{B}(\emptyset)=F_{B}(1)=\emptyset, \quad F_{B}(2)=\{2\}, \quad F_{B}(3)=\{3\} \\
F_{B}(12)=\{1,2\}, \quad F_{B}(13)=\{1,3\}, \quad F_{B}(23)=\{1,2,3\} \\
\bar{F}_{B}(S)=\emptyset \text { for } S \subseteq N .
\end{gathered}
$$

The kernel of $B$ is $\mathcal{K}(B)=\{\{2\},\{3\}\}$.

Moreover, our influence function happens to be the canonical pure influential function of the parents upon the child, that is, $B=B_{23 \rightarrow 1}$. Hence, all players are the followers of the coalition formed by the parents. 


\section{The influence functions}

We turn to influence functions and first make a remark on their structure. $\mathcal{B}$ is the set of mappings from the Boolean lattice $\left(2^{N}, \subseteq\right)$ to $\left(2^{N}, \subseteq\right)$, and so is itself a Boolean lattice. We denote by $\leq$ the order relation, defined as $B \leq B^{\prime}$ iff $B i \leq B^{\prime} i$ for all $i \in I$. Hence $\mathcal{B}$ is atomic, and its atoms are influence functions of the form

$$
B i:= \begin{cases}\left(-1_{N \backslash j}, 1_{j}\right) & \text { if } i=i_{0} \\ -1_{N} & \text { if } i \neq i_{0}\end{cases}
$$

for some $i_{0} \in I$ and $j \in N$. Thus, the number of atoms is $n 2^{n}$. Supremum and infimum are defined by $B \vee B^{\prime}=B \cup B^{\prime}$ and $B \wedge B^{\prime}=B \cap B^{\prime}$.

Next, we define several influence functions $B \in \mathcal{B}$ and investigate their properties, using the tools described in Sections 3 and 4. In particular, for each influence function analyzed, we determine the set of followers and the value of the weighted direct influence index. We do not write down either the sets of the anti-followers or the weighted opposite influence indices anymore, because results are symmetric to the ones concerning the sets of followers and the weighted direct influence indices, and they can be easily determined in an analogous way.

Some simple examples of influence functions are:

(i) The Majority function. Let $n \geq t>\left\lfloor\frac{n}{2}\right\rfloor$, and introduce for any $i \in I$ the set

$$
i^{+}:=\left\{k \in N \mid i_{k}=+1\right\} .
$$

The majority influence function $\mathrm{Maj}^{[t]} \in \mathcal{B}$ is defined by

$$
\operatorname{Maj}^{[t]} i:=\left\{\begin{array}{ll}
1_{N}, & \text { if }\left|i^{+}\right| \geq t \\
-1_{N}, & \text { if }\left|i^{+}\right|<t
\end{array}, \quad \forall i \in I .\right.
$$

If a majority of players has an inclination +1 , then all players decide +1 . If not, all players decide -1 .

(ii) The Guru function. Let $\widetilde{k} \in N$ be a particular player called the guru. The guru influence function Gur $^{[\widetilde{k}]} \in \mathcal{B}$ is defined by

$$
\left(\operatorname{Gur}^{[\widetilde{k}]} i\right)_{j}=i_{\widetilde{k}}, \quad \forall i \in I, \quad \forall j \in N
$$

When a guru exists, every player follows the guru, whatever his inclination.

(iii) The identity function $\mathrm{Id} \in \mathcal{B}$ is defined by

$$
\operatorname{ld} i=i, \quad \forall i \in I
$$

The identity function depicts the absence of any influence.

(iv) The reversal function $-\mathrm{Id} \in \mathcal{B}$ is simply the opposite of the identity function, hence its notation. It depicts a systematic reversal of inclination, but does not show a clear phenomenon of influence. 
(v) The mass psychology effect. Let $t \in(0, n]$ and $\epsilon \in\{-1,+1\}$. Define for any $i \in I$ the set $i^{\epsilon}:=\left\{k \in N \mid i_{k}=\epsilon\right\}$ (remark that $i^{+}=i^{+1}$ ). Functions $B \in \mathcal{B}$ satisfying for each $i \in I$

$$
\text { if }\left|i^{\epsilon}\right| \geq t \text {, then }(B i)^{\epsilon} \supseteq i^{\epsilon}
$$

are called mass psychology influence functions. If there is a sufficiently high number of players with inclination $\epsilon$, it will influence players with a $-\epsilon$ inclination, and some of them will decide $+\epsilon$, while the others will not change. Evidently, the majority function $\mathrm{Maj}^{[t]}$ is of this type with $\epsilon=+1$.

We denote by $\mathcal{B}^{[\epsilon, t]}$ the set of such influence functions. For $\epsilon=-1$, there is an interesting interpretation for functions in $\mathcal{B}^{[-1, t]}$ : such functions depict what could be called the empty restaurant effect, since it is well known that people are reluctant to enter a restaurant with very few people inside.

(vi) Let $t \in(0, n]$. Influence functions in $\mathcal{B}^{[+1, t]} \cap \mathcal{B}^{[-1, n-t+1]}$ are such that for each $i \in I$

$$
i \leq B i \Leftrightarrow\left|i^{+}\right| \geq t, \text { and } i \geq B i \Leftrightarrow\left|i^{+}\right|<t .
$$

These functions also depict a mass psychology effect, but in addition they specify what happens if there is not enough people with inclination +1 : some people with inclination +1 decide for -1 , and the others do not change.

In (3) we have already introduced the notations $I_{S \rightarrow j}^{+}$and $I_{S \rightarrow j}^{-}$for any $\emptyset \neq S \subseteq N$ and $j \in N \backslash S$. More conveniently we introduce $I_{S \rightarrow j}^{\epsilon}:=\left\{i \in I_{S \rightarrow j} \mid i_{S}=\epsilon\right\}$, with $\epsilon \in\{-1,+1\}$, and additionally,

$$
\begin{array}{cc}
I_{S \rightarrow j,>t}^{+}:=\left\{i \in I_{S \rightarrow j}^{+}|| i^{+} \mid>t\right\} & \text { for } t<n-1 \\
I_{S \rightarrow j, \geq t}^{+}:=\left\{i \in I_{S \rightarrow j}^{+}|| i^{+} \mid \geq t\right\} & \text { for } t<n \\
I_{S \rightarrow j,<t}^{-}:=\left\{i \in I_{S \rightarrow j}^{-}|| i^{+} \mid<t\right\} & \text { for } t>1
\end{array}
$$

Similarly, we can replace,+- by $\epsilon$. We list some basic properties of the above mentioned influence functions.

Proposition 4 Let $n \geq t>\left\lfloor\frac{n}{2}\right\rfloor$ and consider the majority function Maj ${ }^{[t]}$. Then the following holds:

(i) For each $\emptyset \neq S \subseteq N$ and $j \in N \backslash S$

$$
d_{\alpha}\left(\mathrm{Maj}^{[t]}, S \rightarrow j\right)= \begin{cases}1, & \text { if } s \geq t \\ \frac{\sum_{i \in I_{S \rightarrow j, \geq t}^{+}} \alpha_{i}^{S \rightarrow j}+\sum_{i \in I_{S \rightarrow j,<t}^{-}} \alpha_{i}^{S \rightarrow j}}{\sum_{i \in I_{S \rightarrow j}} \alpha_{i}^{S \rightarrow j}}, & \text { if } s<t .\end{cases}
$$

If $t=n$, we get $d_{\alpha}\left(\mathrm{Maj}^{[t]}, S \rightarrow j\right)=\frac{1}{2}$.

(ii) For each $S \subseteq N$,

$$
F_{\mathrm{Maj}[t]}(S)= \begin{cases}N, & \text { if } s \geq t \\ \emptyset, & \text { if } s<t .\end{cases}
$$

(iii) The kernel is $\mathcal{K}\left(\mathrm{Maj}^{[t]}\right)=\{S \subseteq N|| S \mid=t\}$. 
Proof: (i) Let $\emptyset \neq S \subseteq N$ be such that $s \geq t$, and $j \in N \backslash S$. If $i_{S}=+1$, then $\left|i^{+}\right| \geq t$ and hence in particular $\left(\mathrm{Maj}^{[t]} i\right)_{j}=+1=i_{S}$. If $i_{S}=-1$, then $\left|i^{+}\right|<t$ and hence in particular $\left(\mathrm{Maj}^{[t]} i\right)_{j}=-1=i_{S}$. This means that $I_{S \rightarrow j}^{*}\left(\mathrm{Maj}^{[t]}\right)=I_{S \rightarrow j}$, and therefore $d_{\alpha}\left(\mathrm{Maj}^{[t]}, S \rightarrow j\right)=1$.

Let $\emptyset \neq S \subseteq N$ be such that $s<t$, and $j \in N \backslash S$. Hence, we get

$$
d_{\alpha}\left(\mathrm{Maj}^{[t]}, S \rightarrow j\right)=\frac{\sum_{i \in I_{S \rightarrow j}^{*}\left(\mathrm{Maj}^{[t]}\right)} \alpha_{i}^{S \rightarrow j}}{\sum_{i \in I_{S \rightarrow j}} \alpha_{i}^{S \rightarrow j}}=\frac{\sum_{i \in I_{S \rightarrow j, \geq t}^{+}} \alpha_{i}^{S \rightarrow j}+\sum_{i \in I_{S \rightarrow j,<t}^{-}} \alpha_{i}^{S \rightarrow j}}{\sum_{i \in I_{S \rightarrow j}} \alpha_{i}^{S \rightarrow j}} .
$$

Let $\emptyset \neq S \subseteq N$ be such that $s<t, t=n$, and $j \in N \backslash S$. Hence, for each $i \in I \backslash\left\{1_{N}\right\}$, $\mathrm{Maj}^{[t]} i=-1_{N}$, and therefore $I_{S \rightarrow j}^{*}\left(\mathrm{Maj}^{[t]}\right)=I_{S \rightarrow j}^{-}$. We have

$$
d_{\alpha}\left(\mathrm{Maj}^{[t]}, S \rightarrow j\right)=\frac{\sum_{i \in I_{S \rightarrow j}^{-}} \alpha_{i}^{S \rightarrow j}}{\sum_{i \in I_{S \rightarrow j}} \alpha_{i}^{S \rightarrow j}}=\frac{\frac{1}{2} \sum_{i \in I_{S \rightarrow j}} \alpha_{i}^{S \rightarrow j}}{\sum_{i \in I_{S \rightarrow j}} \alpha_{i}^{S \rightarrow j}}=\frac{1}{2} .
$$

(ii) Let $S \subseteq N$ be such that $s \geq t$. Suppose that $F_{\mathrm{Maj}[t]}(S) \neq N$. Then there exists $j \in N$ such that $j \notin F_{\mathrm{Maj}^{[t]}}(S)$. Hence, there is $\widetilde{i} \in I_{S}$ such that $\left(\mathrm{Maj}{ }^{[t} \tilde{i}\right)_{j}=-\widetilde{i}_{S}$. Suppose $\widetilde{i}_{S}=+1$. Hence, $\left|i^{+}\right| \geq t$, and therefore for each $k \in N$, (Maj $\left.[t] \widetilde{i}\right)_{k}=+1=\widetilde{i}_{S}$. Suppose then that $\widetilde{i}_{S}=-1$. Hence, $\left|i^{+}\right|<t$, and therefore for each $k \in N$, $\left(\mathrm{Maj}{ }^{[\mathrm{t}]} \tilde{i}\right)_{k}=-1=\widetilde{i}_{S}$, a contradiction again.

Let $S \subseteq N$ be such that $s<t$. Suppose that $F_{\mathrm{Maj}[t]}(S) \neq \emptyset$, and let $\widetilde{j} \in F_{\mathrm{Maj}^{[t]}}(S)$. Hence, for each $i \in I_{S},\left(\mathrm{Maj}^{[t]} i\right)_{\tilde{j}}=i_{S}$. Take $\widetilde{i} \in I_{S}$ such that $\widetilde{i}_{S}=-\widetilde{i}_{k}$ for each $k \notin S$. If $\widetilde{i}_{S}=+1$, then $\left|\widetilde{i}^{+}\right|<t$, and hence for each $j \in N$, (Maj $\left.{ }^{[t} \widetilde{i}\right)_{j}=-1=-\widetilde{i}_{S}$. If $\widetilde{i}_{S}=-1$, then $\left|\widetilde{i}^{+}\right| \geq t$, and hence for each $j \in N$, (Maj $\left.{ }^{[t} \widetilde{i}\right)_{j}=+1=-\widetilde{i}_{S}$, a contradiction.

(iii) By virtue of (8), we have the following. If $|S|<t$, then $F_{\mathrm{Maj}[t]}(S)=\emptyset$, and hence $S \notin \mathcal{K}\left(\mathrm{Maj}{ }^{[t]}\right)$. If $|S|=t$, then $F_{\mathrm{Maj}^{[t]}}(S)=N$, but for each $S^{\prime} \subset S,\left|S^{\prime}\right|<t$, and therefore $F_{\mathrm{Maj}}[t]\left(S^{\prime}\right)=\emptyset$. Hence, $S \in \mathcal{K}\left(\mathrm{Maj}^{[t]}\right)$. If $|S|>t$, then $F_{\mathrm{Maj}^{[t]}}(S)=N$, and there exists $S^{\prime} \subset S$ such that $\left|S^{\prime}\right| \geq t$, which means that $F_{\text {Maj }[t]}\left(S^{\prime}\right)=N$. Hence, $S \notin \mathcal{K}\left(\mathrm{Maj}^{[t]}\right)$.

Note that if the cardinality of a coalition is not smaller than the given threshold $t$, then under the majority function the weighted direct influence index of that coalition on every player outside the coalition is equal to 1, and all players always follow such a coalition. If the cardinality of a coalition is smaller than $t$, then nobody is a follower of that coalition. The kernel of the majority function consists of all the coalitions formed by exactly $t$ players.

Proposition 5 Let $\widetilde{k} \in N$ and consider the guru influence function Gur ${ }^{[\widetilde{k}]}$. Then the following holds:

(i) For each $\emptyset \neq S \subseteq N$ and $j \in N \backslash(S \cup\{\widetilde{k}\})$

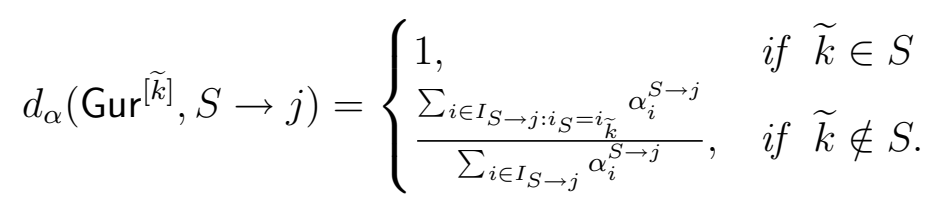

In particular, $\bar{d}\left(\right.$ Gur $\left.^{[\widetilde{k}]}, S \rightarrow j\right)=\frac{1}{2}$ if $\widetilde{k} \notin S$. 
(ii) For each $S \subseteq N$,

$$
F_{\text {Gur }[\tilde{k}]}(S)= \begin{cases}N, & \text { if } \widetilde{k} \in S \\ \emptyset, & \text { if } \widetilde{k} \notin S .\end{cases}
$$

(iii) The kernel is $\mathcal{K}\left(\operatorname{Gur}^{[\tilde{k}]}\right)=\{\widetilde{k}\}$.

(iv) $\operatorname{Gur}^{[\widetilde{k}]}$ is the unique purely influential function of $\widetilde{k}$ upon $N \backslash \widetilde{k}$, i.e., $\mathcal{B}_{\widetilde{k} \rightarrow N \backslash \widetilde{k}}=\left\{\operatorname{Gur}^{[\widetilde{k}]}\right\}$.

Proof: (iv) Gur ${ }^{[\widetilde{k}]} \in \mathcal{B}_{\widetilde{k} \rightarrow N \backslash \widetilde{k}}$ comes immediately from (4) and (5). Now, $\mathcal{B}_{\widetilde{k} \rightarrow N \backslash \widetilde{k}}$ is reduced to a singleton since $I \backslash I_{\widetilde{k}}=\emptyset$.

(i) Let $\emptyset \neq S \subseteq N$ be such that $\widetilde{k} \in S$, and $j \in N \backslash S$. Hence, for each $i \in I,\left(\operatorname{Gur}^{[\widetilde{k}]} i\right)_{j}=i_{\widetilde{k}}$. This means in particular that $I_{S \rightarrow j}^{*}\left(\operatorname{Gur}^{[\widetilde{k}]}\right)=I_{S \rightarrow j}$, and hence $d_{\alpha}\left(\operatorname{Gur}^{[\widetilde{k}]}, S \rightarrow j\right)=1$.

Let $\emptyset \neq S \subseteq N$ be such that $\widetilde{k} \notin S$, and $j \in N \backslash(S \cup\{\widetilde{k}\})$. Hence, if $i \in I_{S \rightarrow j}$ is such that $i_{S}=i_{\widetilde{k}}$, then $i \in I_{S \rightarrow j}^{*}\left(\operatorname{Gur}^{[\widetilde{k}]}\right)$, and if $i \in I_{S \rightarrow j}$ is such that $i_{S}=-i_{\widetilde{k}}$, then $i \notin I_{S \rightarrow j}^{*}\left(\operatorname{Gur}^{[\widetilde{k}]}\right)$. Hence, $\bar{d}\left(\right.$ Gur $\left.^{[\widetilde{k}]}, S \rightarrow j\right)=\frac{1}{2}$.

(ii) The first line can be deduced from the fact that $F_{\text {Gur }}^{[\tilde{k}]}$ is an isotone function and from Prop. 3 (i) since Gur ${ }^{[\widetilde{k}]}$ is purely influential by (iv).

Let $S \subseteq N$ be such that $\widetilde{k} \notin S$. Suppose that $F_{\text {Gur }[\tilde{k}]}(S) \neq \emptyset$. Let $\widetilde{j} \in F_{\text {Gur }[\tilde{k}]}(S)$. Hence, for each $i \in I_{S},\left(\operatorname{Gur}^{[\widetilde{k}]}\right)_{\tilde{j}}=i_{S}$. Take $\widetilde{i} \in I_{S}$ such that $\widetilde{i}_{S}=-\widetilde{i}_{k}$. Hence, for each $j \in N$, $\left(\operatorname{Gur}^{[\widetilde{k}]} \widetilde{i}\right)_{j}=\widetilde{i}_{\widetilde{k}}=-\widetilde{i}_{S}$, and in particular, $\left(\operatorname{Gur}^{[\widetilde{k}]} \widetilde{i}\right)_{\tilde{j}}=\widetilde{i}_{\widetilde{k}}=-\widetilde{i}_{S}$, a contradiction.

(iii) clear from (9).

By virtue of Proposition 5, if the guru belongs to a coalition, then under the guru influence function the weighted direct influence index of that coalition on every player outside the coalition (and not the guru) is equal to 1. Moreover, everybody always follows a coalition with the guru, and nobody is a follower of a coalition without a guru. Only the one-player coalition formed by the guru belongs to the kernel of the guru influence function.

Proposition 6 Let us consider the identity function Id. Then the following holds:

(i) For each $\emptyset \neq S \subseteq N$ and $j \in N \backslash S, d_{\alpha}(\mathrm{Id}, S \rightarrow j)=0$.

(ii) For each $S \subseteq N, F_{\text {ld }}(S)=S$.

(iii) The kernel is $\mathcal{K}(\mathrm{Id})=\{\{k\}, k \in N\}$.

Proof: (i) For each $\emptyset \neq S \subseteq N$ and $j \in N \backslash S, I_{S \rightarrow j}^{*}($ Id $)=\emptyset$, and hence $d_{\alpha}($ Id, $S \rightarrow j)=0$. (ii) Note that $S \subseteq F_{\text {Id }}(S)$, because if $j \in S$, then in particular for each $i \in I_{S},(\operatorname{Id} i)_{j}=$ $i_{j}=i_{S}$. Suppose $F_{\mathrm{ld}}(S) \nsubseteq S$. Hence, there is $k \notin S$ such that $k \in F_{\mathrm{ld}}(S)$, and therefore for each $i \in I_{S},(\operatorname{Id} i)_{k}=i_{S}$. Take $\widetilde{i} \in I_{S}$ such that $\widetilde{i}_{S}=-\widetilde{i}_{k}$. Then we have $(\operatorname{Id} \widetilde{i})_{k}=\widetilde{i}_{S}=-\widetilde{i}_{k}$, but Id $\widetilde{i}=\widetilde{i}$, a contradiction.

(iii) Clear from (ii). 
Note that under the identity function, the weighted direct influence index of an arbitrary coalition on every player outside the coalition is equal to 0. For each coalition, all members of the coalition and only them are the followers of that coalition. The kernel of the identity function consists of all one-player coalitions.

Proposition 7 Let us consider the reversal function - Id. Then the following holds:

(i) For each $\emptyset \neq S \subseteq N$ and $j \in N \backslash S, d_{\alpha}(-\mathrm{ld}, S \rightarrow j)=1$.

(ii) For each $S \subseteq N, F_{- \text {Id }}(S)=\emptyset$.

(iii) The kernel is $\mathcal{K}(-\mathrm{Id})=\emptyset$.

Proof: (i) Take arbitrary $\emptyset \neq S \subseteq N$ and $j \in N \backslash S$. Then $I_{S \rightarrow j}^{*}(-\mathrm{ld})=I_{S \rightarrow j}$, and therefore $d_{\alpha}(-\mathrm{ld}, S \rightarrow j)=1$.

(ii) Suppose there exists $S \subseteq N$ such that $F_{- \text {Id }}(S) \neq \emptyset$, that is, there is $j \in F_{\text {-Id }}(S)$. Then, for each $i \in I_{S},(-\operatorname{ld} i)_{j}=i_{S}$. Take $\widetilde{i} \in I_{S}$ such that $\widetilde{i}_{S}=\widetilde{i}_{j}$. Hence, $(-\operatorname{ld} \widetilde{i})_{j}=\widetilde{i}_{S}=\widetilde{i}_{j}$, but $-\mathrm{Id} \widetilde{i}=-\widetilde{i}$, a contradiction.

(iii) It comes immediately from (ii).

Under the reversal function, the weighted direct influence index of an arbitrary coalition on every player outside the coalition is equal to 1 . Moreover, the kernel of the reversal function and the sets of followers for every coalition are empty.

Proposition 8 Let $t \in(0, n]$ and $\epsilon \in\{-1,+1\}$ be fixed, and consider any influence function $B$ in $\mathcal{B}^{[\epsilon, t]}$. Then the following holds:

(i) There exists $B \in \mathcal{B}^{[\epsilon, t]}$ such that for each $\emptyset \neq S \subseteq N$ and $j \in N \backslash S$,

$$
d_{\alpha}(B, S \rightarrow j)=0
$$

(ii) If $t=1$, then for each $\emptyset \neq S \subseteq N, j \in N \backslash S$,

$$
d_{\alpha}(B, S \rightarrow j) \leq \frac{1}{2}
$$

Moreover, the upper bound $\frac{1}{2}$ is attained for at least one function $B$ in $\mathcal{B}^{[\epsilon, t]}$.

(iii) For each $\emptyset \neq S \subseteq N$ such that $s>n-t, t>1$, and $j \in N \backslash S$, there exists $B \in \mathcal{B}^{[\epsilon, t]}$ such that

$$
d_{\alpha}(B, S \rightarrow j)=1
$$

(iv) For each $\emptyset \neq S \subseteq N$ such that $s \leq n-t, t>1, j \in N \backslash S$,

$$
d_{\alpha}(B, S \rightarrow j) \leq \frac{\sum_{i \in I_{S \rightarrow j}^{\epsilon}} \alpha_{i}^{S \rightarrow j}+\sum_{i \in I_{S \rightarrow j,<t}^{-\epsilon}} \alpha_{i}^{S \rightarrow j}}{\sum_{i \in I_{S \rightarrow j}} \alpha_{i}^{S \rightarrow j}} .
$$

In particular,

$$
\bar{d}(B, S \rightarrow j) \leq \frac{1}{2}+\frac{1}{2^{n-s}} \sum_{p=0}^{t-2}\left(\begin{array}{c}
n-s-1 \\
p
\end{array}\right)
$$




$$
\underline{d}(B, S \rightarrow j) \in\left\{0, \frac{1}{2}\right\} .
$$

Moreover, for each $\emptyset \neq S \subseteq N$ such that $s \leq n-t$ and $j \in N \backslash S$, there is $B \in \mathcal{B}^{[\epsilon, t]}$ such that

$$
d_{\alpha}(B, S \rightarrow j)=\frac{\sum_{i \in I_{S \rightarrow j}^{\epsilon}} \alpha_{i}^{S \rightarrow j}+\sum_{i \in I_{S \rightarrow j,<t}^{-\epsilon}} \alpha_{i}^{S \rightarrow j}}{\sum_{i \in I_{S \rightarrow j}} \alpha_{i}^{S \rightarrow j}} .
$$

(v) For each $S \subseteq N$,

$$
F_{B}(S) \subseteq S \text { if } s \leq n-t .
$$

Moreover, there exists $B \in \mathcal{B}^{[\epsilon, t]}$ such that for each $S \subseteq N, F_{B}(S)=S$.

Proof: (i) Remark that $\mathrm{Id} \in \mathcal{B}^{[\epsilon, t]}$ for any $\epsilon \in\{-1,+1\}$ and any $t \in(0, n]$. By virtue of Proposition 6 , (i), we get $d_{\alpha}$ (ld, $\left.S \rightarrow j\right)=0$ for each $\emptyset \neq S \subseteq N$ and $j \in N \backslash S$.

(ii) Let $t=1$. For each $\emptyset \neq S \subseteq N$ and $j \in N \backslash S$, we have, using (2)

$$
\sum_{i \in I_{S \rightarrow j}} \alpha_{i}^{S \rightarrow j}=2 \sum_{i \in I_{S \rightarrow j}^{\epsilon}} \alpha_{i}^{S \rightarrow j}
$$

In addition, $I_{S \rightarrow j}^{*}(B) \cap I_{S \rightarrow j}^{-\epsilon}=\emptyset$. Hence,

$$
d_{\alpha}(B, S \rightarrow j)=\frac{\sum_{i \in I_{S \rightarrow j}^{*}(B)} \alpha_{i}^{S \rightarrow j}}{\sum_{i \in I_{S \rightarrow j}} \alpha_{i}^{S \rightarrow j}} \leq \frac{\sum_{i \in I_{S \rightarrow j}^{\epsilon}(B)} \alpha_{i}^{S \rightarrow j}}{\sum_{i \in I_{S \rightarrow j}} \alpha_{i}^{S \rightarrow j}}=\frac{1}{2} .
$$

Remark that $B$ defined by $B i=\epsilon 1_{N}$ for each $i \in I$ belongs to $\mathcal{B}^{[\epsilon, 1]}$. Take arbitrary $\emptyset \neq S \subseteq N$ and $j \in N \backslash S$. Note that $I_{S \rightarrow j}^{*}(B)=I_{S \rightarrow j}^{\epsilon}$. Hence, for this $B$

$$
d_{\alpha}(B, S \rightarrow j)=\frac{1}{2}
$$

(iii) Take arbitrary $\emptyset \neq S \subseteq N$ such that $s>n-t, t>1$, and $j \notin S$. Define $B$ as follows:

$$
(B i)_{k}= \begin{cases}i_{S}, & \text { if } i \in I_{S \rightarrow j} \text { and } k=j \\ i_{k}, & \text { otherwise }\end{cases}
$$

Note that $B$ belongs to $\mathcal{B}^{[\epsilon, t]}$, because if $i \in I_{S \rightarrow j}^{-\epsilon}$, then $\left|i^{\epsilon}\right|<t$, and if $i \in I \backslash I_{S \rightarrow j}^{-\epsilon}$, then (6) is satisfied. We have $d_{\alpha}(B, S \rightarrow j)=1$.

(iv) Let $B \in \mathcal{B}^{[\epsilon, t]}$. Take arbitrary $\emptyset \neq S \subseteq N$ such that $s \leq n-t, t>1$, and $j \notin S$. Note that if $i \in I_{S \rightarrow j}^{-\epsilon}$ is such that $\left|i^{\epsilon}\right| \geq t$, then $i \notin I_{S \rightarrow j}^{*}(B)$. Hence,

$$
d_{\alpha}(B, S \rightarrow j) \leq \frac{\sum_{i \in I_{S \rightarrow j}^{\epsilon}} \alpha_{i}^{S \rightarrow j}+\sum_{i \in I_{S \rightarrow j,<t}^{-\epsilon}} \alpha_{i}^{S \rightarrow j}}{\sum_{i \in I_{S \rightarrow j}} \alpha_{i}^{S \rightarrow j}}
$$

Moreover,

$\bar{d}(B, S \rightarrow j)=\frac{\left|I_{S \rightarrow j}^{*}(B)\right|}{2^{n-s}} \leq \frac{\left|I_{S \rightarrow j}^{\epsilon}\right|}{2^{n-s}}+\frac{1}{2^{n-s}} \sum_{p=0}^{t-2}\left(\begin{array}{c}n-s-1 \\ p\end{array}\right)=\frac{1}{2}+\frac{1}{2^{n-s}} \sum_{p=0}^{t-2}\left(\begin{array}{c}n-s-1 \\ p\end{array}\right)$ 
Moreover, $\underline{d}(B, S \rightarrow j) \neq 1$, because if $i \in I_{S \rightarrow j}^{-\epsilon}$ is such that $i_{k}=\epsilon$ for each $k \notin S$, then $i \notin I_{S \rightarrow j}^{*}(B)$. Hence, $\underline{d}(B, S \rightarrow j) \in\left\{0, \frac{1}{2}\right\}$.

Finally, define $B$ as follows. For each $k \neq j,(B i)_{k}=i_{k}$ for $i \in I$, and

$$
(B i)_{j}= \begin{cases}\epsilon, & \text { if } i \in I_{S \rightarrow j}^{\epsilon} \\ -\epsilon, & \text { if } i \in I_{S \rightarrow j}^{-\epsilon} \text { and }\left|i^{\epsilon}\right|<t \\ i_{j}, & \text { otherwise }\end{cases}
$$

This $B$ belongs to $\mathcal{B}^{[\epsilon, t]}$, and we get then

$$
d_{\alpha}(B, S \rightarrow j)=\frac{\sum_{i \in I_{S \rightarrow j}^{\epsilon}} \alpha_{i}^{S \rightarrow j}+\sum_{i \in I_{S \rightarrow j,<t}^{-\epsilon}} \alpha_{i}^{S \rightarrow j}}{\sum_{i \in I_{S \rightarrow j}} \alpha_{i}^{S \rightarrow j}} .
$$

(v) Let $t \in(0, n]$ and $s \leq n-t$. Suppose there is $B \in \mathcal{B}^{[\epsilon, t]}$ such that $F_{B}(S) \nsubseteq S$ for a certain $S \subseteq N$. This means that $F_{B}(S) \neq \emptyset$, since $\emptyset \subseteq S$ for each $S$. Hence, there is $k \notin S$ such that $k \in F_{B}(S)$. This means that for each $i \in I_{S},(B i)_{k}=i_{S}$. Take $\tilde{i} \in I_{S}$ such that $\widetilde{i}_{S}=-\epsilon, \widetilde{i}_{k}=\epsilon$, and $\left|\widetilde{i}^{\epsilon}\right| \geq t$. Such an $\widetilde{i}$ always exists, because $n-s \geq t$. We have $(\widetilde{B i})_{k}=\widetilde{i}_{S}=-\epsilon$, and therefore $\left.\widetilde{(i}\right)^{\epsilon} \nsubseteq(\widetilde{i i})^{\epsilon}$. But since $\left|\widetilde{i^{\epsilon}}\right| \geq t$, we should have $(\widetilde{i})^{\epsilon} \subseteq(\widetilde{B i})^{\epsilon}$, a contradiction.

If we take $B=\mathrm{Id}$, then for each $S \subseteq N, F_{\mathrm{ld}}(S)=S$.

Proposition 9 Let $B \in \mathcal{B}^{[+1, t]} \cap \mathcal{B}^{[-1, n-t+1]}$, i.e., satisfying (7). Then the following holds:

(i) For each $t \in(0, n]$ there exists $B \in \mathcal{B}^{[+1, t]} \cap \mathcal{B}^{[-1, n-t+1]}$ such that for each $\emptyset \neq S \subseteq N$ and $j \in N \backslash S$,

$$
\bar{d}(B, S \rightarrow j)=0 .
$$

(ii) If $t \in\{1, n\}$, then for each $\emptyset \neq S \subseteq N, j \in N \backslash S$,

$$
d_{\alpha}(B, S \rightarrow j) \leq \frac{1}{2}
$$

Moreover, there exists $B \in \mathcal{B}^{[+1, t]} \cap \mathcal{B}^{[-1, n-t+1]}$ such that for each $\emptyset \neq S \subseteq N$ and $j \in N \backslash S$,

$$
d_{\alpha}(B, S \rightarrow j)=\frac{1}{2} \text {. }
$$

(iii) For each $\emptyset \neq S \subseteq N$ such that $s \geq t>n-s, 1<t<n$, and $j \in N \backslash S$, there exists $B \in \mathcal{B}^{[+1, t]} \cap \mathcal{B}^{[-1, n-t+1]}$ such that

$$
d_{\alpha}(B, S \rightarrow j)=1
$$

(iv) For each $\emptyset \neq S \subseteq N$ such that $s \leq n-t, 1<t \leq n, j \in N \backslash S$,

$$
d_{\alpha}(B, S \rightarrow j) \leq \frac{\sum_{i \in I_{S \rightarrow j}^{+}} \alpha_{i}^{S \rightarrow j}+\sum_{i \in I_{S \rightarrow j,<t}^{-}} \alpha_{i}^{S \rightarrow j}}{\sum_{i \in I_{S \rightarrow j}} \alpha_{i}^{S \rightarrow j}}
$$


the upper bound being attained for at least one $B$ in $\mathcal{B}^{[+1, t]} \cap \mathcal{B}^{[-1, n-t+1]}$. In particular,

$$
\begin{gathered}
\bar{d}(B, S \rightarrow j) \leq \frac{1}{2}+\frac{1}{2^{n-s}} \sum_{p=0}^{t-2}\left(\begin{array}{c}
n-s-1 \\
p
\end{array}\right) \\
\underline{d}(B, S \rightarrow j) \in\left\{0, \frac{1}{2}\right\} .
\end{gathered}
$$

(v) For each $\emptyset \neq S \subseteq N$ such that $s<t, t<n$, and $j \in N \backslash S$

$$
d_{\alpha}(B, S \rightarrow j) \leq \frac{\sum_{i \in I_{S \rightarrow j}^{-}} \alpha_{i}^{S \rightarrow j}+\sum_{i \in I_{S \rightarrow j, \geq t}^{+}} \alpha_{i}^{S \rightarrow j}}{\sum_{i \in I_{S \rightarrow j}} \alpha_{i}^{S \rightarrow j}},
$$

the upper bound being attained for at least one $B$ in $\mathcal{B}^{[+1, t]} \cap \mathcal{B}^{[-1, n-t+1]}$. In particular,

$$
\begin{gathered}
\bar{d}(B, S \rightarrow j) \leq \frac{1}{2}+\frac{1}{2^{n-s}} \sum_{p=t-s}^{n-s-1}\left(\begin{array}{c}
n-s-1 \\
p
\end{array}\right) \\
\underline{d}(B, S \rightarrow j) \in\left\{0, \frac{1}{2}\right\} .
\end{gathered}
$$

(vi) For each $\emptyset \neq S \subseteq N$ such that $s<t \leq n-s, 1<t<n, j \in N \backslash S$,

$$
d_{\alpha}(B, S \rightarrow j) \leq \frac{\sum_{i \in I_{S \rightarrow j, \geq t}^{+}} \alpha_{i}^{S \rightarrow j}+\sum_{i \in I_{S \rightarrow j,<t}^{-}} \alpha_{i}^{S \rightarrow j}}{\sum_{i \in I_{S \rightarrow j}} \alpha_{i}^{S \rightarrow j}},
$$

the upper bound being attained for at least one $B$ in $\mathcal{B}^{[+1, t]} \cap \mathcal{B}^{[-1, n-t+1]}$. In particular,

$$
\begin{gathered}
\bar{d}(B, S \rightarrow j) \leq \frac{1}{2^{n-s}} \sum_{p=t-s}^{n-s-1}\left(\begin{array}{c}
n-s-1 \\
p
\end{array}\right)+\frac{1}{2^{n-s}} \sum_{p=0}^{t-2}\left(\begin{array}{c}
n-s-1 \\
p
\end{array}\right) \\
\underline{d}(B, S \rightarrow j)=0 .
\end{gathered}
$$

(vii) For each $S \subseteq N$,

$$
\begin{aligned}
& F_{B}(S) \subseteq S \text { if } s \leq n-t \text { or } s<t \\
& F_{B}(S) \supseteq S \text { if } n-s<t \leq s .
\end{aligned}
$$

Moreover, there is $B \in \mathcal{B}$ such that for each $S \subseteq N, F_{B}(S)=S$.

Proof: (i), (ii) and (iii): just apply Proposition 8 for $\mathcal{B}^{[+1, t]}$ and $\mathcal{B}^{[-1, n-t+1]}$.

(iv) and (v) correspond to (iv) in Proposition 8 for $\epsilon=+1,-1$ respectively. Then (vi) is intersection of both.

(vii) The first line comes from Proposition $8(\mathrm{v})$.

Let $s \geq t>n-s$. Suppose there is $B \in \mathcal{B}^{[+1, t]} \cap \mathcal{B}^{[-1, n-t+1]}$ such that $S \nsubseteq F_{B}(S)$ for a certain $S \subseteq N$. This means that there exists $k \in S$ such that $k \notin F_{B}(S)$, and therefore there is $\widetilde{i} \in I_{S}$ such that $(\widetilde{B i})_{k}=-\widetilde{i}_{S}$. If $\widetilde{i}_{S}=+1$, then $(\widetilde{B i})_{k}=-1$, and hence $\widetilde{i} \not \subset B \widetilde{i}$. But since $s \geq t$, we have $\left|i^{+}\right| \geq t$, and therefore $\widetilde{i} \leq \widetilde{B i}$. Hence, $\widetilde{i}_{S}=-1$, and $(\widetilde{B i})_{k}=+1$. But this means that $\widetilde{i} \geq \widetilde{B i}$. Since $t>n-s$, we have $\left|i^{+}\right|<t$, and therefore $\widetilde{i} \geq \widetilde{B i}$, a contradiction.

If we take $B=\mathrm{ld}$, then for each $S \subseteq N, F_{B}(S)=S$. 


\section{The modified decisional power}

After studying the influence, we consider the second part of our description, namely the group decision part. We analyze the concepts of Success, Decisiveness, Luck, and Failure of a player, which are defined as a probability that the player is successful, is decisive, is lucky, and fails, respectively. In Rusinowska and De Swart (2006), when the two functions $B$ and $g d$ were not separated, the notions of Success, Luck, Failure, and Decisiveness of a player were defined starting not from the final decision of the player in question, but from his inclination (for an analysis of success and decisiveness of a player in voting situations, see for instance Laruelle and Valenciano (2005)). Consequently, based on the definition given in Rusinowska and De Swart (2006), we say that a player is successful if his inclination coincides with the group decision. When we adopt such a definition of being successful, if all inclination vectors are equally probable, then the generalized HoedeBakker index is a kind of a 'net' Success (Rusinowska, 2008), i.e., it is equal to 'Success - Failure $=$ Decisiveness'. Moreover, under such a definition of Success, if all inclination vectors are equally probable, then the generalized Hoede-Bakker index coincides with the absolute Banzhaf index; see Rusinowska and De Swart (2006).

If we separate the two functions $B$ and $g d$, we can define Success, Failure, Luck, and Decisiveness of a player starting from the final decision of the player, not as before from the inclination. For instance, under such a definition, a player is said to be successful if his decision coincides with the group decision. This is equivalent to the definition of success in a voting situation, presented in Laruelle and Valenciano (2005), where a player is said to be successful if his vote coincides with the result (i.e., if either the player votes YES and the proposal is accepted, or he votes NO and the proposal is rejected).

In our framework of players' decisions and the group decision, we can formalize the concepts of success and decisiveness as follows. Given a probability distribution $p: I \rightarrow$ $[0,1]$ over all inclination vectors, and $B \in \mathcal{B}$, we define $p_{B}=P \circ B^{-1}$ on $I$ (probability of the decision vectors), where $P$ is the probability measure induced by $p$. We define now the group decision function $g d: I \rightarrow\{+1,-1\}$ on the set of all $n$-vectors, assigning (as before) the value +1 if the group decision is YES, and -1 if the group decision is NO. Moreover, for $b \in I$ and $k \in N$, we define $b^{-k} \in I$ by

$$
b_{j}^{-k}=\left\{\begin{array}{cc}
b_{j} & \text { if } j \neq k \\
-b_{j} & \text { if } j=k
\end{array}\right.
$$

Definition 8 Given $g d \in \mathcal{G}, p_{B}: I \rightarrow[0,1]$, we define for each $k \in N$

- Player k's Success

$$
\operatorname{SUC}_{k}\left(g d, p_{B}\right):=\operatorname{Pr}(k \text { is successful })=\sum_{\left\{b \in I \mid b_{k}=g d(b)\right\}} p_{B}(b)
$$

- Player k's Failure

$$
\operatorname{FAIL}_{k}\left(g d, p_{B}\right):=\operatorname{Pr}(k \text { fails })=\sum_{\left\{b \in I \mid b_{k}=-g d(b)\right\}} p_{B}(b)
$$

- Player k's Decisiveness

$$
\operatorname{DEC}_{k}\left(g d, p_{B}\right):=\operatorname{Pr}(k \text { is decisive })=\sum_{\left\{b \in I \mid b_{k}=g d(b)=-g d\left(b^{-k}\right)\right\}} p_{B}(b)
$$


- Player k's Luck

$$
\operatorname{LUCK}_{k}\left(g d, p_{B}\right):=\operatorname{Pr}(k \text { is lucky })=\sum_{\left\{b \in I \mid b_{k}=g d(b)=g d\left(b^{-k}\right)\right\}} p_{B}(b)
$$

According to Barry (1980), the following relation between Success, Luck, and Decisiveness holds:

$$
\text { Success }=\text { Decisiveness }+ \text { Luck }
$$

and in our case, we have for each $k \in N, p_{B}$, and $g d \in \mathcal{G}$

$$
\begin{gathered}
\mathrm{SUC}_{k}\left(g d, p_{B}\right)=\mathrm{DEC}_{k}\left(g d, p_{B}\right)+\operatorname{LUCK}_{k}\left(g d, p_{B}\right) \\
\operatorname{SUC}_{k}\left(g d, p_{B}\right)=1-\mathrm{FAIL}_{k}\left(g d, p_{B}\right) .
\end{gathered}
$$

Based on the above, we modify the generalized Hoede-Bakker index in two respects. Fist of all, the inclination vectors do not need to be equally probable, as it was assumed in the original framework. Moreover, we do not consider the inclination vectors with the positive (or negative) inclination of the given player, but we consider the inclination vectors that lead to the positive (or negative) decision of the player in question. We propose therefore the following modification of the decisional power:

Definition 9 Given $B \in \mathcal{B}, g d \in \mathcal{G}, p: I \rightarrow[0,1]$, we define for each $k \in N$ the modified decisional power by

$$
\phi_{k}(B, g d, p):=\sum_{\left\{i \mid(B i)_{k}=+1\right\}} p(i) g d(B i)-\sum_{\left\{i \mid(B i)_{k}=-1\right\}} p(i) g d(B i) .
$$

The modified decisional power is also a kind of the net success, that is, we have the following:

Proposition 10 Given $B \in \mathcal{B}, g d \in \mathcal{G}$, and $p: I \rightarrow[0,1]$, for each $k \in N$

$$
\phi_{k}(B, g d, p)=\mathrm{SUC}_{k}\left(g d, p_{B}\right)-\mathrm{FAIL}_{k}\left(g d, p_{B}\right) .
$$

Proof: Let $B \in \mathcal{B}, g d \in \mathcal{G}, p: I \rightarrow[0,1]$, and $k \in N$. From (12), we have

$$
\begin{aligned}
\phi_{k}(B, g d, p) & =\sum_{\left\{i \mid(B i)_{k}=+1\right\}} p(i) g d(B i)-\sum_{\left\{i \mid(B i)_{k}=-1\right\}} p(i) g d(B i) \\
& =\sum_{\left\{i \mid(B i)_{k}=g d(B i)\right\}} p(i)-\sum_{\left\{i \mid(B i)_{k}=-g d(B i)\right\}} p(i)
\end{aligned}
$$

By virtue of (10) and (11),

$$
\begin{aligned}
\operatorname{SUC}_{k}\left(g d, p_{B}\right)-\mathrm{FAIL}_{k}\left(g d, p_{B}\right) & =\sum_{\left\{b \in I \mid b_{k}=g d(b)\right\}} p_{B}(b)-\sum_{\left\{b \in I \mid b_{k}=-g d(b)\right\}} p_{B}(b) \\
& =\sum_{\left\{b \in B(I) \mid b_{k}=g d(b)\right\}} \sum_{\{i \mid B i=b\}} p(i)-\sum_{\left\{b \in B(I) \mid b_{k}=-g d(b)\right\}} \sum_{\{i \mid B i=b\}} p(i) \\
& =\sum_{\left\{i \mid(B i)_{k}=g d(B i)\right\}} p(i)-\sum_{\left\{i \mid(B i)_{k}=-g d(B i)\right\}} p(i)=\phi_{k}(B, g d, p)
\end{aligned}
$$


Let us notice that while $\phi$ is equal to 'Success - Failure' under the new definition of being successful (based on decisions, not as before on inclinations), in general it is not equal to 'Decisiveness' anymore.

Example 3 Let us come back to the Family Example. Since the weather happens to be rather unstable, the actors are not that enthusiastic to decide for the trip. Moreover, a new attractive computer game, a romance just bought and looking very interesting, and a telecast of an important football match are of importance when making the decision. The inclinations of the players to say YES are independent of each other and their probabilities are equal to $\frac{1}{2}, \frac{1}{3}$, and 0 , for the child, the mother and the father, respectively. The parents try not to discriminate their child in family decision-making, and it is agreed that the family decides for the trip if at least two family members say YES. Table 2 presents the probability distribution over all inclination vectors, and the decision vectors, while Table 3 shows the probability distribution over all decision vectors, and the group decisions.

Table 2. The inclination and decision vectors

\begin{tabular}{c|c|c|c|c|c|c|c|c}
$i \in I$ & $(1,1,1)$ & $(1,1,-1)$ & $(1,-1,1)$ & $(-1,1,1)$ & $(1,-1,-1)$ & $(-1,1,-1)$ & $(-1,-1,1)$ & $(-1,-1,-1)$ \\
\hline$p(i)$ & 0 & $\frac{1}{6}$ & 0 & 0 & $\frac{1}{3}$ & $\frac{1}{6}$ & 0 & $\frac{1}{3}$ \\
\hline$B(i)$ & $(1,1,1)$ & $(1,1,-1)$ & $(1,-1,1)$ & $(1,1,1)$ & $(-1,-1,-1)$ & $(-1,1,-1)$ & $(-1,-1,1)$ & $(-1,-1,-1)$
\end{tabular}

Table 3. The group decision

\begin{tabular}{c|c|c|c|c|c|c}
$b \in B(I)$ & $(1,1,1)$ & $(1,1,-1)$ & $(1,-1,1)$ & $(-1,1,-1)$ & $(-1,-1,1)$ & $(-1,-1,-1)$ \\
\hline$p_{B}(b)$ & 0 & $\frac{1}{6}$ & 0 & $\frac{1}{6}$ & 0 & $\frac{2}{3}$ \\
\hline$g d(b)$ & +1 & +1 & +1 & -1 & -1 & -1
\end{tabular}

Moreover, $g d(-1,1,1)=+1$, and $g d(1,-1,-1)=-1$.

Based on the given information, we get the following:

$$
\begin{gathered}
\operatorname{SUC}_{1}\left(g d, p_{B}\right)=1, \quad \operatorname{SUC}_{2}\left(g d, p_{B}\right)=\operatorname{SUC}_{3}\left(g d, p_{B}\right)=\frac{5}{6} \\
\mathrm{FAIL}_{1}\left(g d, p_{B}\right)=0, \quad \mathrm{FAIL}_{2}\left(g d, p_{B}\right)=\mathrm{FAIL}_{3}\left(g d, p_{B}\right)=\frac{1}{6} \\
\mathrm{DEC}_{1}\left(g d, p_{B}\right)=\frac{1}{3}, \quad \mathrm{DEC}_{2}\left(g d, p_{B}\right)=\mathrm{DEC}_{3}\left(g d, p_{B}\right)=\frac{1}{6} \\
\mathrm{LUCK}_{1}\left(g d, p_{B}\right)=\mathrm{LUCK}_{2}\left(g d, p_{B}\right)=\mathrm{LUCK}_{3}\left(g d, p_{B}\right)=\frac{2}{3} \\
\phi_{1}(B, g d, p)=1, \quad \phi_{2}(B, g d, p)=\phi_{3}(B, g d, p)=\frac{2}{3} .
\end{gathered}
$$




\section{Conclusions}

The improvement brought in this paper emphasizes the role of the influence function in the Hoede-Bakker index. In order to analyze the influence in this framework, the two parts, i.e., the influence part and the group decision part, are separated. The description proposed here fully takes into account the mutual influence among players. In particular, we define the possibility influence index which takes into account any possibility of influence, and the certainty influence index which expresses certainty of influence. These indices are special cases of the weighted influence index, where some weights of influence of a coalition are introduced. These weights depend on the number of players that have the same inclination as the coalition in question. We also distinguish between the direct influence and the opposite influence. Both concepts express the ability of a coalition to make a player vote differently from his inclination. When influencing directly, the inclinations of the player and the coalition are different from each other, and the decision of the player coincides with the inclination of the coalition. When calculating the opposite influence, we consider the inclination vectors such that the inclinations of the player and the coalition coincide, but the decision of the player is different from the inclination of the coalition.

Why do not we introduce just one influence index? The answer is simple. We cannot do it, because the process of influence is very complex and one may imagine many possible kinds of influence. In order to capture (most or all of) them, we define the general tool, the weighted (direct or opposite) influence index which, depending on the assumptions and weights, can measure the different kinds of influence. To the best of our knowledge, such influence indices have not been proposed before, and seem to be a very useful tool, in particular, in the theory of coalition and alliance formation, negotiations, and more generally multi-agent systems.

Apart from the influence indices, we investigate other tools to analyze the influence and the influence functions, like the set of followers of a coalition, the concept of the purely influential function, and the kernel of an influence function. All these tools help us to understand and characterize better the process of influence. In particular, we study properties of several interesting 'real-world' influence functions.

To summarize, in the paper we first separate the two parts of the model. Since the influence part of the framework has not been studied before in the literature, we focus on this first part of the model. We try to analyze what happens in the process of influence, i.e., we look at the link between an (original) inclination of a player and his (final) decision. In particular, we investigate the influence functions and we propose the indices to measure the influence. This is presented in Sections 3, 4, and 5, and constitutes the core of the paper. Nevertheless, in order to obtain the whole picture of the model, we analyze also the second part of the framework, i.e., the process from the decisions of the agents to the group decision. This is presented in Section 6. Since the group decision part is related to voting situations that have been considered before, we first link our framework to a probabilistic model presented in the voting power literature. First of all, for the second part of our model we define the concepts of success, failure, decisiveness, and luck. These concepts link the (final) decisions of the agents to the group decision. Moreover, we propose a modified decisional power, which allows the inclination vectors to be unequally probable, and which is based on the decisions and the group decision. It is important to 
stress that we do not consider the new definition of success and decisiveness to be better than the one given in Rusinowska and De Swart (2006), where the concepts of success and decisiveness link the (original) inclinations of the agents to the group decision. These approaches are simply different from each other. If, for example, we consider a successful player as a player whose decision coincides with the group decision, then the approach of the present paper should be applied. However, if a successful player is rather seen as a player whose inclination coincides with the group decision, which seems to be quite natural as well, then the 'old' approach should be used.

There are still several other improvements we would like to bring to this framework in our future research. One of the new research lines may be to introduce dynamic aspects. The framework analyzed here is, in fact, a decision process after a single step of mutual influence. In reality, the mutual influence does not stop necessarily after one step but may iterate. We propose to study the behavior of the series $B i, B^{2} i, \ldots, B^{n} i$; to find convergence conditions, and to study the corresponding decisional power. An interesting generalization would be also to assume that the influence function $B$ is a probabilistic function. Another improvement could be to enlarge the set of possible decisions to games on product lattices (Grabisch and Lange, 2007). Moreover, we would like to provide an axiomatic characterization of the influence indices. Finally, in order to get a deeper insight into the process of influence between agents, some experiments on this issue could be conducted.

\section{References}

1. Banzhaf, J., 1965. Weighted voting doesn't work: a mathematical analysis. Rutgers Law Review 19, 317-343.

2. Barry, B., 1980. Is it better to be powerful or lucky?, Part I and Part II. Political Studies 28, 183-194, 338-352.

3. Coleman, J.S., 1971. Control of collectivities and the power of a collectivity to act. In: Lieberman, B., (Ed.), Social Choice. New York: Gordon and Breach, pp. 269-300.

4. Coleman, J.S., 1986. Individual Interests and Collective Action: Selected Essays, Cambridge University Press.

5. Deegan, J., Packel, E.W., 1978. A new index of power for simple n-person games. International Journal of Game Theory 7, 113-123.

6. Dubey, P., Shapley, L.S., 1979. Mathematical properties of the Banzhaf power index. Mathematics of Operations Research 4, 99-131.

7. Feix, M., Lepelley, D., Merlin, V., Rouet, J.-L., 2007. On the voting power of an alliance and the subsequent power of its members. Social Choice and Welfare 28, 181-207.

8. Felsenthal, D., Machover, M., 1998. The Measurement of Voting Power: Theory and Practice, Problems and Paradoxes, London: Edward Elgar Publishers.

9. Felsenthal, D., Machover, M., 2002. Annexations and alliances: when are blocs advantageous a priori? Social Choice and Welfare 19, 295-312.

10. Felsenthal, D., Machover, M., Zwicker, W., 1998. The bicameral postulates and indices of a priori voting power. Theory and Decision 44, 83-116.

11. M. Grabisch and F. Lange, 2007. Games on lattices, multichoice games and the Shapley value: a new approach. Mathematical Methods of Operations Research, 65, 153-167.

12. Hoede, C., Bakker, R., 1982. A theory of decisional power. Journal of Mathematical Sociology 8, 309-322.

13. Holler, M.J., Packel, E.W., 1983. Power, luck and the right index. Journal of Economics 43, 21-29.

14. Hu, X., Shapley, L.S., 2003. On authority distributions in organizations: equilibrium. Games and Economic Behavior 45, 132-152.

15. Johnston, R.J., 1978. On the measurement of power: some reactions to Laver. Environment and Planning A 10, 907-914.

16. Laruelle, A., Valenciano, F., 2005. Assessing success and decisiveness in voting situations. Social Choice and Welfare 24, 171-197.

17. Owen, G., 1977. Values of games with a priori unions. In: Hein, R., Moeschlin, O. (Eds.), Essays in Mathematical Economics and Game Theory. New York: Springer-Verlag, pp. 77-88. 
18. Owen, G., 1981. Modification of the Banzhaf-Coleman index for games with a priori unions. In: Holler, M.J. (Ed.), Power, Voting, and Voting Power. Würzburg-Wien: Physica Verlag, pp. 232-238.

19. Penrose, L.S., 1946. The elementary statistics of majority voting. Journal of the Royal Statistical Society 109, 53-57.

20. Rae, D., 1969. Decision-rules and individual values in constitutional choice. American Political Science Review 63, 40-56.

21. Rusinowska, A., 2008. On the not-preference-based Hoede-Bakker index. Forthcoming in: Petrosjan, L.A., and Mazalov, V.V. (Eds.), Game Theory and Applications. Vol. XIII, Nova Science Publishers, Inc. New York.

22. Rusinowska, A., De Swart, H., 2006. Generalizing and modifying the Hoede-Bakker index. In: De Swart, H., et al. (Eds.), Theory and Applications of Relational Structures as Knowledge Instruments. Springer's Lecture Notes in Artificial Intelligence, LNAI 4342, Springer, Heidelberg, Germany, pp. 60-88.

23. Shapley, L.S., 1953. A value for n-person games. Annals of Mathematics Studies 28, 307-317.

24. Shapley, L.S., Shubik, M., 1954. A method for evaluating the distribution of power in a committee system. American Political Science Review 48, 787-792.

25. van den Brink, R., Borm, P., 2002. Digraph competitions and cooperative games. Theory and Decision 53 , 327-342.

26. van den Brink, R., Gilles, R.P., 2000. Measuring domination in directed networks. Social Networks 22, 141157.

27. van den Brink, R., van der Laan, G., 1998. Axiomatization of the normalized Banzhaf value and the Shapley value. Social Choice and Welfare 15, 567-582.

28. van der Laan, G., van den Brink, R., 1998. Axiomatization of a class of share functions for n-person games. Theory and Decision 44, 117-148.

29. van der Laan, G., van den Brink, R., 2002. A Banzhaf share function for cooperative games in coalition structure. Theory and Decision 53, 61-86.

30. van den Brink, R., Borm, P., Hendrickx, R., Owen, G., 2007. Characterizations of the $\beta$ - and the degree network power measure, Theory and Decision (forthcoming). 